\title{
Espécies raras e comuns de Myrtaceae da Floresta Estacional Decidual de Santa Catarina, Brasil
}

\author{
Rare and common Myrtaceae species in Santa Catarina Seasonal Deciduous forest, \\ Brazil
}

Cláudia Fontana ${ }^{1,4}$, André Luís de Gasper ${ }^{2}$ \& Lúcia Sevegnani ${ }^{3}$

\begin{abstract}
Resumo
Objetivou-se avaliar as espécies arbóreas raras e comuns da família Myrtaceae amostradas pelo Inventário Florístico Florestal de Santa Catarina na Floresta Estacional Decidual. Esta floresta ocorre ao longo do rio Uruguai e afluentes, sob baixas temperaturas no inverno e chuvas bem distribuídas ao longo do ano, com árvores do dossel e emergentes predominantemente caducifólias. Coletaram-se dados em 78 unidades amostrais com 0,40 ha cada, registrando-se árvores com diâmetro na altura do peito $\geq 10 \mathrm{~cm}$. Utilizou-se a metodologia Rabinowitz para segregar as espécies em raras ou comuns, considerando três variáveis: distribuição geográfica, especificidade por habitat e tamanho populacional. Das 28 espécies amostradas, 78,57\% (22 spp.) apresentaram alguma forma de raridade e 21,43\% (6 spp.) foram comuns. As espécies de Myrtaceae na Floresta Estacional Decidual são, em geral, amplamente distribuídas, mas seletivas quanto ao habitat e possuem poucos indivíduos. Destacam-se três espécies consideradas raras na floresta avaliada: Eugenia subterminalis, Myrcianthes gigantea e Siphoneugena reitzii com um indivíduo cada. Campomanesia xanthocarpa foi a espécie mais comum. Todas as espécies avaliadas são zoocóricas e podem ser utilizadas em restauração ambiental.
\end{abstract}

Palavras-chave: domínio Mata Atlântica, análise de raridade, conservação.

\begin{abstract}
We surveyed the common and rare species of Myrtaceae that were sampled in the Santa Catarina Forest Floristic Inventory of the Seasonal Deciduous Forest. This forest runs along the Uruguay River and its tributaries, with cold winters and rainfall distributed throughout the year, and a mostly deciduous and emergent canopy. We collected data in 78 sample units, each with an area of 0.40 ha, and recorded trees $\geq 10 \mathrm{~cm}$ diameter at breast height. We used Rabinowitz methodology to separate common and rare species, based on three variables: geographic distribution, habitat and population size. Of the 28 species sampled, 78.57\% (22 spp.) were rare and $21.43 \%$ (6 spp.) were common. Myrtaceae species in the Seasonal Deciduous Forest are generally widely distributed, but are selective in relation to habitat and low in abundance. It is important to note that three species were considered rare in this forest: Eugenia subterminalis DC., Myrcianthes gigantea (D.Legrand) D.Legrand and Siphoneugena reitzii D.Legrand with one individual each. Campomanesia xanthocarpa (Mart.) O.Berg was the most common species. All species are dispersed by animals and can be used in habitat restoration.

Key words: Atlantic Forest domain, rarity analysis, conservation.
\end{abstract}

\section{Introdução}

O termo raro é definido de diversas formas entre os pesquisadores (Rabinowitz et al. 1986; Martins 1991; Gaston 1994). No contexto de biologia de populações e comunidades, é habitualmente empregado no sentido de restrito (Caiafa \& Martins 2010), sendo caracterizadas como raras as espécies endêmicas e com pequeno tamanho populacional (Gaston 1994). Para a ecologia a raridade pode apresentar gradientes, variando entre casos extremos e mais brandos de raridade; Rabinowitz et al. (1986) desenvolveram metodologias para segregar formas de raridade, bem como as espécies ditas comuns, tomando

\footnotetext{
${ }^{1}$ Universidade Regional de Blumenau, R. São Paulo 3366, Itoupava Seca, 89030-000, Blumenau, SC, Brasil.

${ }^{2}$ Universidade Regional de Blumenau, R. Antônio da Veiga 140, Victor Konder, 89012-900, Blumenau, SC, Brasil.

${ }^{3}$ Universidade Regional de Blumenau, R. São Paulo 3366, Itoupava Seca, 89030-000, Blumenau, SC, Brasil.

${ }^{4}$ Autor para correspondência: claudia@metodosistemas.com.br
} 
por base três variáveis compartilhadas entre as espécies: distribuição geográfica, especificidade por habitat e tamanho populacional. A metodologia proposta por Rabinowitz et al. (1986) para análise de raridade tem sido utilizada mundialmente por vários pesquisadores, como Pitman et al. (1999), que analisaram a raridade na floresta amazônica peruana, Broennimann et al. (2005), que pesquisaram a raridade na Suíça e Drever et al. (2010) o fizeram no nordeste de Ontário, Canadá. No Brasil existem até o presente momento os estudos realizados por Caiafa \& Martins (2010) para a porção meridional da Floresta Ombrófila Densa e por Fontana (2012) para a Floresta Estacional Decidual de Santa Catarina. Pitman et al. (1999) defendem que essa metodologia continua sendo uma das melhores para tratar a raridade das espécies em multiescala e de forma compreensível. O conhecimento dos gradientes de raridade é importante, pois possibilita distinguir onde devem ocorrer ações prioritárias de conservação.

Myrtaceae compreende cerca de 144 gêneros e 5.774 espécies no mundo (The Plant List 2010), abrangendo árvores e arbustos que se distribuem em diversos habitats e por todos os continentes, com exceção da Antártica (Marchiori \& Sobral 1997), sendo de distribuição. No Brasil ocorrem 979 espécies e 23 gêneros (Sobral et al. 2012), sendo estes tipicamente neotropicais, com exceção de Eugenia que é pantropical (Landrum \& Kawasaki 1997; Marchiori \& Sobral 1997). As espécies nativas brasileiras geralmente não produzem madeiras valiosas, no entanto, muitas espécies são frutíferas, algumas de elevada importância comercial, sendo apreciadas pela fauna e pelas pessoas (Marchiori \& Sobral 1997; Backes \& Irgang 2004; Carvalho 2006, 2008, 2010).

No Sul do Brasil, Myrtaceae frequentemente tem sido relatada com grande riqueza específica (Klein 1972; Marchiori \& Sobral 1997; Bergamin \& Bodin 2006; Chaves et al. 2009; Kilka \& Longui 2011). Porém, conforme dados da Flora Ilustrada Catarinense (Legrand \& Klein 1967, 1969, 1970, 1972, 1977, 1978), há um grande número de espécies que são frequentemente raras ou com inexpressiva dispersão, principalmente na Floresta Estacional Decidual (FED). Apesar de sua importância na estrutura das florestas, estudos ecológicos abordando especificamente mirtáceas sul-brasileiras ainda são escassos, principalmente para o Estado de Santa Catarina, em especial na FED. Isto gera preocupação quanto à conservação destas, uma vez que a FED em Santa Catarina está sob grande pressão de uso (Klein 1978; Vibrans et al. 2008; Vibrans et al. 2012b). Conforme Landrum \& Kawasaki (1997) espécies de mirtáceas estão desaparecendo da natureza antes mesmo que se tenha conhecimento básico de sua biologia, o que justifica a análise de raridade das espécies catarinenses.

Caiafa \& Martins (2010) na categorização das formas de raridade da porção Meridional da Floresta Ombrófila Densa, constataram que Myrtaceae destacou-se como a família com maior riqueza de espécies e Fontana (2012), para a FED em Santa Catarina, como a segunda família com maior riqueza, atrás apenas de Fabaceae. Para avaliar as espécies de Myrtaceae presentes na FED catarinense, foi utilizada a lista apresentada por Fontana (2012), tendo como hipótese que as espécies de Myrtaceae ocorrem de forma muito rara nesta região fitoecológica, considerando a alta interferência antropogênica relatada para esta floresta e a grande importância desta família na fitofisionomia da Mata Atlântica. Espera-se que os resultados possam subsidiar ações de conservação das espécies de Myrtaceae presentes nesta região fitoecológica, bem como os ecossistemas que as contêm.

\section{Material e Métodos}

O presente trabalho utilizou os dados coletados em campo pelo Inventário Florístico Florestal de Santa Catarina (IFFSC), levantados no âmbito da Floresta Estacional Decidual. A Floresta Estacional faz parte do domínio da Mata Atlântica, que em Santa Catarina distribui-se ao longo do rio Uruguai e seus afluentes (Klein 1978), entre a longitude $51^{\circ} 07^{\prime} 19^{\prime \prime} \mathrm{W}$ e $53^{\circ} 46^{\prime} 34^{\prime \prime} \mathrm{W}$. Conforme a classificação de Koeppen (1948) o clima predominante é "mesotérmico úmido com verão quente" ( $\mathrm{Cfa})$, com temperaturas médias entre $17^{\circ} \mathrm{C}$ e $19^{\circ} \mathrm{C}$ e ocorrência de geadas de 5 a 20/dias por ano (Nimer 1971). As chuvas são bem distribuídas (Leite \& Klein 1990), com precipitação média entre $1.500 \mathrm{~mm}$ a $2.250 \mathrm{~mm}$ (Nimer 1971). No Sul do Brasil, diferentemente de outras regiões do país, o caráter estacional da vegetação, que provoca a queda das folhas, é dado em função das baixas temperaturas no inverno e não em razão de períodos de estiagem (Oliveira-Filho et al. 2006; Schumacker et al. 2011).

Para alocar as unidades amostrais (UAs) na FED foi utilizada uma amostragem sistemática, 
com UAs distribuídas em grades de $5 \mathrm{~km} \times 5$ $\mathrm{km}$, que resultou em 78 UAs para esta região fitoecológica (Vibrans et al. 2010). Cada UA foi composta por um conglomerado básico com área total de $4.000 \mathrm{~m}^{2}$, totalizando 31,2 hectares, onde foram amostrados todos os indivíduos com DAP $\geq 10 \mathrm{~cm}$ (Vibrans et al. 2010).

Para avaliar as formas de raridade de Myrtaceae ocorrentes na FED, a partir dos dados coletados nas 78 unidades amostrais do IFFSC nesta região, foi utilizada a listagem de espécies compilada por Fontana (2012), resgatando-se as informações sobre a categorização da raridade das espécies de mirtáceas. Para elaboração da lista utilizou-se a grade de parâmetros proposta por Rabinowitz et al. (1986) e empregada no Brasil por Caiafa \& Martins (2010), gerando uma matriz com oito categorias, uma contendo espécies comuns e as outras sete abrigando as formas de raridade (Tab. 1). Esse sistema de classificação partiu de três variáveis comuns entre as espécies, a distribuição geográfica, a especificidade por habitat e o tamanho populacional. O gradiente crescente de raridade vai de 1 a 7 , sendo que a Forma 7 é considerada a mais severa pelos autores do método (Rabinowitz et al. 1986).

Para encontrar as espécies pertencentes a cada forma de raridade, bem como as comuns, aplicou-se a grade de parâmetros com os critérios de enquadramento apresentados na Tabela 1.

Para a variável distribuição geográfica, utilizou-se o gradiente longitudinal em toda a extensão de ocorrência da FED no Estado, pois, diferentemente de Caiafa \& Martins (2010), que analisaram a latitude, uma vez que a Mata Atlântica (sensu stricto) acompanha a faixa litorânea e abrange grandes latitudes, a FED se estende pelo interior catarinense em estreita latitude, mas ocupando ampla faixa longitudinal (Fig. 1), o que torna esta análise mais pertinente. A longitude foi analisada a cada $30^{\prime}$ ou meio grau, equivalendo a $55 \mathrm{~km}$. Desta forma, as UAs do IFFSC (2010), perfazem seis gradientes possíveis nessa região fitoecológica. A classificação em "ampla distribuição geográfica" foi aplicada à espécie ocorrente em três ou mais gradientes longitudinais, podendo cobrir extensão superior a $165 \mathrm{~km}$. Para a classificação como "restrita distribuição geográfica" foi considerada a ocorrência da espécie em dois ou apenas um gradiente longitudinal, ou seja, presente em uma extensão máxima de $110 \mathrm{~km}$.

Para a variável especificidade por habitat considerou-se o gradiente de altitude ocorrente nesta região fitoecológica, especificado pelo Instituto Brasileiro de Geografia e Estatística (IBGE 1992; Vibrans et al. 2011), sendo que a formação Submontana situa-se entre as latitudes $24^{\circ} \mathrm{S}$ a $32^{\circ} \mathrm{S}$ e altitudes de $30 \mathrm{~m}$ a $400 \mathrm{~m}$ e a formação Montana situa-se entre as latitudes $24^{\circ} \mathrm{S}$ a $32^{\circ} \mathrm{S}$ e as altitudes de $400 \mathrm{~m}$ a $1000 \mathrm{~m}$. Em Santa Catarina, a FED apresenta as formações Submontana e Montana (neste estudo considerado para categorizar o habitat), nas latitudes compreendida entre $27^{\circ} 08^{\prime} 07^{\prime \prime S}$ e $27^{\circ} 48^{\prime} 39^{\prime \prime}$ 'S e com altitudes de 250 a 898 m (Vibrans et al. 2012a), embora seja conhecido que dentro de cada faixa altitudinal os fatores ambientais podem variar consideravelmente. Portanto, foi considerada como tendo habitat único aquela espécie que se apresentou em apenas uma formação e com habitat variado, a espécie ocorrente nas duas formações.

Para a variável tamanho populacional considerou-se o número de indivíduos amostrados em cada UA. Dessa forma, a espécie que apresentou apenas um indivíduo nas UA em que ocorreu, foi considerada escassa. Considerou-se abundante a espécie que esteve presente, em pelo menos uma das UA em que ocorreu, com dois ou mais indivíduos. Portanto, com a aplicação destes critérios foi possível segregar as espécies desta família em cinco formas de raridade, além das consideradas comuns.

Tabela 1 - Grade de parâmetros para classificação das formas de raridade.

Table 1 - Grid parameters for classifying forms of rarities.

\begin{tabular}{|c|c|c|c|c|c|}
\hline \multirow{2}{*}{\multicolumn{2}{|c|}{$\begin{array}{l}\text { Distribuição geográfica } \\
\text { Especificidade de habitat }\end{array}$}} & \multicolumn{2}{|c|}{ Ampla } & \multicolumn{2}{|c|}{ Restrita } \\
\hline & & Variado & Único & Variado & Único \\
\hline \multirow{2}{*}{$\begin{array}{l}\text { Tamanho } \\
\text { populacional }\end{array}$} & Abundantes & Espécies Comuns & Forma 2 & Forma 4 & Forma 6 \\
\hline & Escassas & Forma 1 & Forma 3 & Forma 5 & Forma 7 \\
\hline
\end{tabular}

Fonte: Adaptado de Rabinowtiz et al. (1986) e Caiafa \& Martins (2010). 


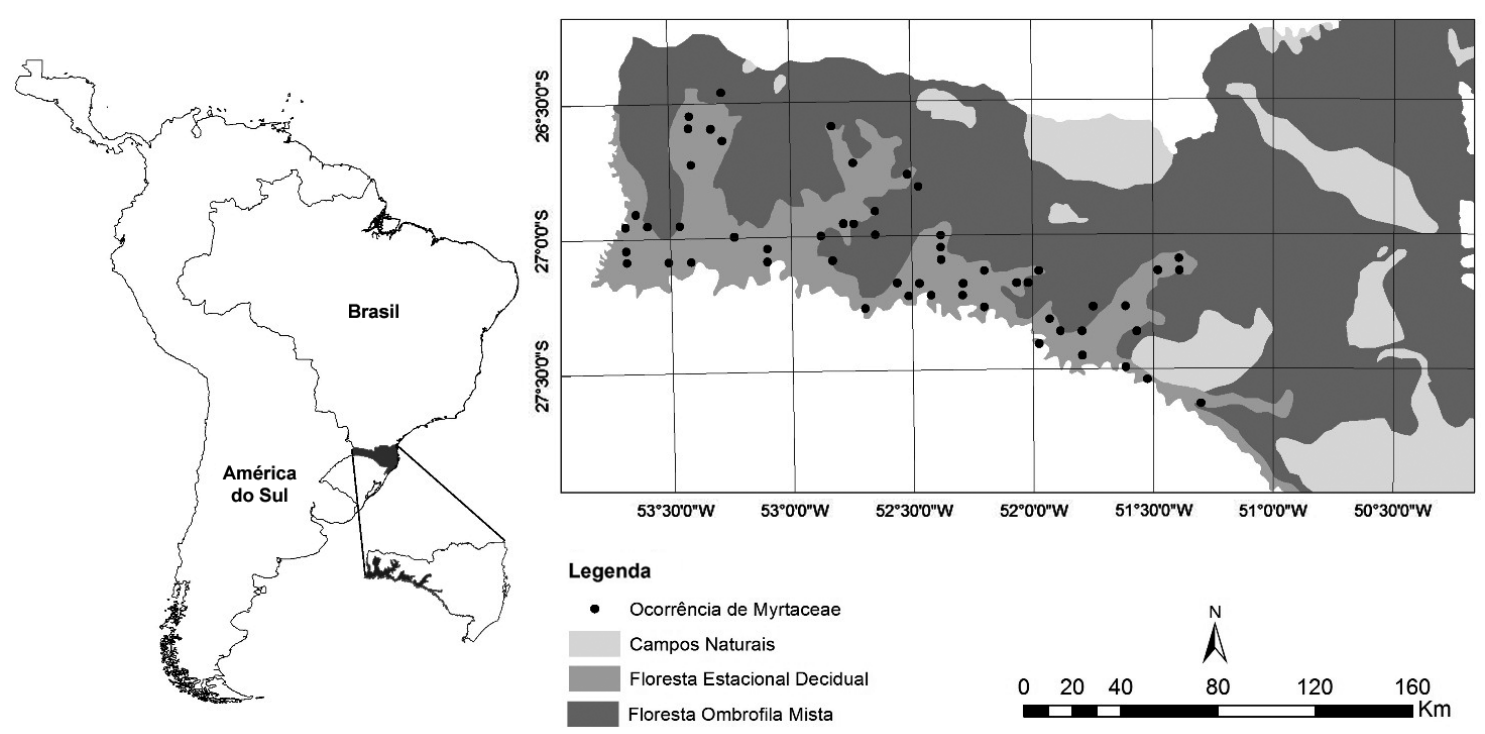

Figura 1 - Distribuição da família Myrtaceae na região fitoecológica da Floresta Estacional Decidual em Santa Catarina. Figure 1 - Distribution of the family Myrtaceae in the region phytoecological Deciduous Forest in Santa Catarina.

\section{Resultados e Discussão}

Foram avaliados 337 indivíduos, representando 28 espécies, agrupadas em 10 gêneros de Myrtaceae na FED catarinense (Tab. 3). Esta família está amplamente distribuída na região estudada (Fig. 1). O gênero mais representativo foi Eugenia L., com 10 espécies (39,29\%), seguido de Myrcia DC. com quatro (14,29\%), Campomanesia Ruiz \& Pav. com três (10,71\%), Calyptranthes Sw., Myrcianthes O.Berg e Plinia L. com três espécies cada $(7,14 \%)$, Blepharocalyx O.Berg, Myrceugenia O.Berg, Myrciaria O.Berg e Siphoneugena O.Berg com uma espécie cada $(3,57 \%)$.

Conforme Romagnolo \& Souza (2006), o gênero Eugenia é bem representado nas diversas formações vegetacionais do Brasil, apresentando elevada riqueza específica, com espécies frequentes e abundantes, sendo que este gênero abrange cerca de 241 espécies no domínio atlântico, das quais 202 são endêmicas (Stehmann et al. 2009). Segundo Chaves et al. (2009) Eugenia, conjuntamente com Myrcia, compreendem o maior número de espécies nativas dessa família, conferindo importante papel nas florestas brasileiras. A riqueza destes dois gêneros na FED catarinense sugere que, apesar da intensa interferência antropogênica (Vibrans et al. 2008), os fragmentos florestais mantém a proporção destas espécies nesta floresta.

Do total das espécies de Myrtaceae amostradas $78,57 \%$ (22 spp.) apresentam alguma forma de raridade (Formas 2, 3, 5, 6 e 7), as Formas 1 (espécies amplamente distribuídas, com habitat variado, mas escassas) e 4 (espécies restritamente distribuídas, mas em habitat variado e abundantes) não ocorrem, e 21,43\% (6 spp.) são comuns (Tab. 2). Conforme Ricklefs (2011), nas florestas tropicais poucas espécies são abundantes e muitas mais são raras.

As espécies foram preferencialmente estenotópicas (Dajoz 1983), ou seja, com restrita distribuição na área estudada (Formas 5, 6 e 7), abrangendo 67,86\% (20) (Tab. 2). Esse resultado evidencia o caráter endêmico das espécies das Florestas Estacionais relatado por Pennington et al. (2009). São espécies com especificidade por habitat ou estenóicas, $75 \%$ (21 spp.) delas, portanto, com restrição quanto ao habitat (Formas 2, 3, 6 e 7), ou seja, foram registradas em apenas uma das formações (Montana ou Submontana) (Tab. 2). Este resultado pode indicar baixa tolerância frente à variação das condições ambientais (Dajoz 1983), pois com a altitude variam também os fatores climáticos.

Quanto ao tamanho populacional, 53,47\% (15 espécies) foram enquadradas como escassas e 46,43\% (13 espécies) como abundantes na região (Tab. 2). As características de distribuição e abundância encontradas para as Myrtaceae da FED catarinense corroboram as descrições feitas por Legrand \& Klein $(1967,1969,1970,1972,1977$, 1978), os quais relataram que a maior parte das espécies desta família são raras ou com distribuição 
Tabela 2 - Distribuição das espécies nas formas de raridade, segundo critérios propostos por Rabinowitz et al. (1986), encontradas na Floresta Estacional Decidual em Santa Catarina.

Table 2 - Distribution of species in the forms of rarity, according to the criteria proposed by Rabinowitz et al. (1986), found in the Deciduous Forest in Santa Catarina.

\begin{tabular}{|c|c|c|c|c|c|}
\hline \multicolumn{2}{|c|}{ Distribuição geográfica } & \multicolumn{2}{|c|}{$\begin{array}{l}\text { Ampla } \\
32,14 \%\end{array}$} & \multicolumn{2}{|c|}{$\begin{array}{c}\text { Restrita } \\
67,86 \%\end{array}$} \\
\hline & cificidade de habitat & Variado & Único & Variado & Único \\
\hline \multirow{2}{*}{$\begin{array}{l}\text { Tamanho } \\
\text { populacional }\end{array}$} & $\begin{array}{l}\text { Abundantes } \\
\text { (Algumas amostras com } \\
\text { muitos indivíduos) 46,43\% }\end{array}$ & $\begin{array}{c}\text { Espécies comuns } \\
21,43 \%(6)\end{array}$ & $\begin{array}{c}\text { Forma } 2 \\
3,57 \%(1)\end{array}$ & $\begin{array}{c}\text { Forma } 4 \\
0\end{array}$ & $\begin{array}{c}\text { Forma } 6 \\
21,43 \%(6)\end{array}$ \\
\hline & $\begin{array}{l}\text { Escassas } \\
\text { (Todas as amostras um } \\
\text { indivíduo) } 53,57 \%\end{array}$ & $\begin{array}{c}\text { Forma } 1 \\
0\end{array}$ & $\begin{array}{c}\text { Forma } 3 \\
7,14 \%(2)\end{array}$ & $\begin{array}{c}\text { Forma } 5 \\
3,57 \%(1)\end{array}$ & $\begin{array}{c}\text { Forma } 7 \\
42,86 \%(12)\end{array}$ \\
\hline
\end{tabular}

descontínua e inexpressiva. O mesmo foi observado na série de livros recentemente publicados pelo Inventário Florístico Florestal de Santa Catarina, onde constam as análises da abundância de cada uma das espécies amostradas (Vibrans et al. 2012a,b, 2013a,b). Conforme Izco (1998) a baixa abundância local é condicionante da raridade e pode preceder a extinção local.

As espécies categorizadas como Formas 2 e 3 de raridade, são compostas por arranjos com ocorrência em ampla extensão e podem ser menos prioritárias em termos de conservação da natureza (Izco 1998). Porém, considerando que esta fitofisionomia é a mais ameaçada do domínio atlântico e que existe apenas uma unidade de conservação que a proteje em território catarinense, o Parque Estadual Fritz Plaumann, localizado na bacia hidrográfica do rio do Peixe, altamente impactada pela agroindústria, onde alterações do meio interferem na unidade de conservação empobrecendo gradativamente sua diversidade biológica (Vitali \& Uhlig 2010), as populações destas espécies devem ser monitoradas com objetivo de evitar extinções locais.

A análise das espécies comuns evidenciou que das seis espécies presentes na FED, todas ocorrem também na Floresta Ombrófila Mista (FOM) e duas são igualmente comuns na porção meridional da Floresta Ombrófila Densa (FOD) (Legrand \& Klein 1967, 1969, 1970, 1972, 1977, 1978; Caiafa \& Martins 2010; Vibrans et al. 2011), sendo as últimas Campomanesia guazumifolia (Cambess.) O.Berg e C. xanthocarpa (Mart.) O.Berg. No entanto, Caiafa \& Martins (2010) demostraram que outras duas comuns na FED catarinense apresentaram raridade na FOD: Eugenia ramboi D. Legrand (F4) e E. uniflora L. (F1) (Tab. 3). Dentre as espécies categorizadas como comuns destacam- se Calyptranthes tricona D.Legrand e Myrcianthes pungens $(\mathrm{O}$. Berg) D. Legrand, que foram relatadas como raras em Santa Catarina e Campomanesia guazumifolia e Eugenia uniflora relatadas como raras apenas na FED (Legrand \& Klein 1969, 1977). A espécie mais abundante foi Campomanesia xanthocarpa, também relatada como comum na FED (Legrand \& Klein 1977) e na porção meridional da FOD (Caiafa \& Martins 2010) (Tab. 3).

Todas as 12 espécies incluídas na Forma 7 , a mais restritiva, foram também encontradas nos estudos de Legrand \& Klein (1967, 1969, 1970, 1972, 1977, 1978). A Forma 7 corresponde às espécies com restrita distribuição geográfica, seletivas quanto ao habitat e com escassos indivíduos. Das espécies assim categorizadas Campomanesia guaviroba, Eugenia uruguayensis, E. brasiliensis e E. subterminalis foram consideradas muito raras por Legrand \& Klein $(1969,1972,1977)$, e Myrcianthes gigantea rara apenas na FED (Legrand \& Klein 1977). As quatro primeiras também estão presentes na porção meridional da FOD (Caiafa \& Martins 2010) (Tab. 3). Legrand \& Klein (1969) descreveram Eugenia burkartiana como característica de FOD, mas possível de ser encontrada na FED, uma vez que ocorre também no Paraguai e Missiones (Argentina) e, de fato, no presente estudo foi coletada na Estacional. Essa espécie foi relatada como a forma mais rara na porção meridional da FOD (F7) (Caiafa \& Martins 2010), porém, está presente nas outras regiões fitoecológicas florestais de Santa Catarina (Tab. 3). Legrand \& Klein (1978) citam Myrciaria floribunda como sinônimo heterotípico de Myrciaria arborea D.Legrand, Myrciaria axillaris O.Berg, Myrciaria ciliolata (Cambess.) O.Berg, Myrciaria splendens O.Berg e Myrciaria tenuiramis O.Berg. Esses sinônimos foram incluídos posteriormente na sinonímia de Myrciaria floribunda (ver Sobral et al. 
Tabela 3 - Categorização das espécies de Myrtaceae (APG III 2009) distribuídas nas formas de raridades e as classificadas como comuns. Registro na FOM - Floresta Ombrófila Mista, FOD - Floresta Ombrófila Densa, na publicação FIC - Flora Ilustrada Catarinense e classificação encontrada por Caiafa \& Martins (CM) para porção meridional da Floresta Ombrófila Densa. $\mathrm{N}=$ Indica o número de indivíduos; $\mathrm{C}=$ Indica as espécies comuns na porção meridional da Floresta Ombrófila Densa (Caiafa \& Martins 2010); CM = Indica as formas de raridade (1, 4, 5, 6 e 7) na porção meridional da Floresta Ombrófila Densa (Caiafa \& Martins 2010); × = Indica a presença das espécies na Floresta Ombrófila Densa em Santa Catarina (Vibrans et al. 2011), Floresta Ombrófila Mista (Vibrans et al. 2011) e constantes na Flora Ilustrada Catarinense (Legrand \& Klein 1967-1978).

Table 3 - Categorization of species of Myrtaceae (APG III 2009) distributed in the forms of rarities and classified as common. Log in FOM - Araucaria Forest, FOD - Rain Forest, published in FIC - Illustrated Flora Catarinense and classification found by Caiafa and Martins (CM) for the southern portion of the Atlantic Rain Forest. $\mathrm{N}=$ Indicates the number of individuals; $\mathrm{C}=$ Indicates species common in the southern portion of the Rain Forest (Caiafa \& Martins 2010); CM = Indicates forms of rarity (1, 4, 5, 6 and 7) in the southern portion of the Rain Forest (Caiafa \& Martins 2010); $\times$ = Indicates the presence of the species in the Atlantic Rain Forest in Santa Catarina (Vibrans et al. 2011), Rain Forest (Vibrans et al. 2011) and listed in the Illustrated Flora of Santa Catarina (Legrand \& Klein 1967, 1969, 1970, 1972, 1977, 1978).

\begin{tabular}{|c|c|c|c|c|c|c|}
\hline Espécie & Forma & $\mathbf{N}$ & $\mathbf{C M}$ & FOD & FOM & FIC \\
\hline Blepharocalyx salicifolius (Kunth) O.Berg & Forma 7 & 2 & $\mathrm{C}$ & $\times$ & $\times$ & $\times$ \\
\hline Calyptranthes grandifolia O.Berg & Forma 7 & 1 & F4 & $\times$ & $\times$ & $\times$ \\
\hline C. tricona D. Legrand & Comum & 19 & & $\times$ & $\times$ & $\times$ \\
\hline Campomanesia guaviroba (DC.) Kiaersk. & Forma 7 & 1 & $\mathrm{C}$ & $\times$ & & $\times$ \\
\hline C. guazumifolia (Cambess.) O.Berg & Comum & 27 & $\mathrm{C}$ & $\times$ & $\times$ & $\times$ \\
\hline C. xanthocarpa (Mart.) O.Berg & Comum & 88 & $\mathrm{C}$ & $\times$ & $\times$ & $\times$ \\
\hline Eugenia brasiliensis Lam. & Forma 7 & 1 & $\mathrm{C}$ & $\times$ & & $\times$ \\
\hline E. burkartiana (D. Legrand) D. Legrand & Forma 7 & 1 & F7 & $\times$ & $\times$ & $\times$ \\
\hline E. gracillima Kiaersk. & Forma 6 & 6 & & & & \\
\hline E. involucrata DC. & Forma 3 & 5 & $\mathrm{C}$ & $\times$ & $\times$ & $\times$ \\
\hline E. pyriformis Cambess. & Forma 3 & 6 & F6 & & $\times$ & $\times$ \\
\hline E. ramboi D. Legrand & Comum & 6 & $\mathrm{~F} 4$ & $\times$ & $\times$ & $\times$ \\
\hline E. rostrifolia D. Legrand & Forma 2 & 63 & F4 & $\times$ & $\times$ & $\times$ \\
\hline E. subterminalis DC. & Forma 7 & 1 & & & $\times$ & $\times$ \\
\hline E. uniflora L. & Comum & 48 & $\mathrm{~F} 1$ & & $\times$ & $\times$ \\
\hline E. uruguayensis Cambess. & Forma 7 & 1 & F4 & & $\times$ & $\times$ \\
\hline E. verticillata (Vell.) Angely & Forma 6 & 12 & & $\times$ & $\times$ & $\times$ \\
\hline Myrceugenia myrcioides (Cambess.) O. Berg & Forma 7 & 2 & $\mathrm{C}$ & $\times$ & $\times$ & $\times$ \\
\hline Myrcia catharinensis (D.Legrand) NicLugh. & Forma 6 & 7 & & $\times$ & $\times$ & $\times$ \\
\hline M. oblongata DC. & Forma 6 & 3 & & & $\times$ & $\times$ \\
\hline M. pubipetala Miq. & Forma 7 & 1 & $\mathrm{C}$ & $\times$ & & $\times$ \\
\hline M. splendens (Sw.) DC. & Forma 6 & 2 & & $\times$ & $\times$ & $\times$ \\
\hline Myrcianthes gigantea (D. Legrand) D. Legrand & Forma 7 & 1 & & & $\times$ & $\times$ \\
\hline M. pungens (O. Berg) D. Legrand & Comum & 24 & & & $\times$ & $\times$ \\
\hline Myrciaria floribunda (H. West ex Willd.) O. B & Forma 7 & 3 & $\mathrm{C}$ & $\times$ & $\times$ & $\times$ \\
\hline Plinia peruviana (Poir.) Govaerts & Forma 5 & 3 & F5 & $\times$ & $\times$ & $\times$ \\
\hline P. rivularis (Cambess.) Rotman & Forma 6 & 2 & $\mathrm{C}$ & $\times$ & $\times$ & $\times$ \\
\hline Siphoneugena reitzii $\mathrm{D}$. Legrand & Forma 7 & 1 & & $\times$ & $\times$ & $\times$ \\
\hline
\end{tabular}


2013). Alguns desses sinônimos são citados como raros (principalmente na FED) e outros frequentes ou abundantes (principalmente na vertente atlântica), concluindo-se que a espécie foi comum no estado, especialmente na FOD. Os trabalhos recentes do IFFSC confirmam a predominância da espécie na FOD, onde foram amostrados 36 indivíduos (Vibrans et al. 2013a) e menor presença para a FOM, com 14 indivíduos amostrados (Vibrans et al. 2013b). Desse modo, é possível constatar que ela é rara apenas na FED catarinense, onde se amostrou apenas três indivíduos (Tab. 3), uma vez que foi comum também na porção meridional da FOD (Caiafa \& Martins 2010).

As espécies que compõem a Forma 7 de raridade estão distribuídas em 12 UAs e estas dispõem-se, em sua maioria, concentradas nas bacias dos rios Jacutinga e Irani (Fig. 2). Esse resultado indica áreas importantes para conservação de conjuntos de espécies raras de Mytaceae nessa região.

As espécies categorizadas na Forma 6 de raridade têm distribuição geográfica restrita e seletividade por habitat, no entanto, ocorrem com maior número de indivíduos, sendo consideradas pela metodologia como abundante. Das seis espécies que compõem esta forma, apenas Plinia rivularis foi relatada para a porção meridional da FOD (Tab. 3). Quatro delas foram encontradas nas duas outras regiões fitoecológicas florestais do Estado: Eugenia verticillata, Myrcia catharinensis, M. splendens e Plinia rivularis (Tab. 3). Myrcia oblongata além da FED, está presente apenas na FOM (Tab. 3), e foi relatada como de ocorrência exclusiva no planalto, sendo importante na expansão da floresta sobre os campos naturais, preparando terreno para as demais espécies, onde pode formar capões, como dominante (Legrand \& Klein 1969). A partir do registro desta espécie na FED pode-se inferir sobre uma possível dispersão dela também nesta região fitoecológica. A espécie Eugenia gracillima destaca-se por não ter sido relatada em nenhuma das listas consultadas, porém, na Lista de Espécies da Flora do Brasil (Sobral et al. 2013) consta sua ocorrência para a Bahia e os três estados do Sul do Brasil. No livro dedicado à Floresta Estacional Subtropical também não foi encontrado registro para ela (Schumacher et al. 2011). Segundo Lughadha et al. (2012), Myrcia catharinensis ocorre nos estados do Sul do Brasil.

Apenas Plinia peruviana foi categorizada na Forma 5 de raridade e ocorreu com igual forma nos estudos de Caiafa \& Martins (2010) para a porção meridional da FOD no Brasil (Tab. 3).

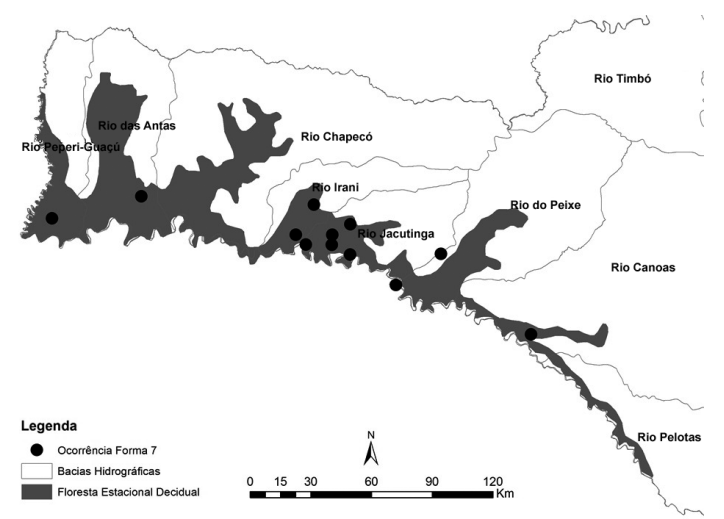

Figura 2 - Distribuição da Forma 7 de raridade de espécies da família Myrtaceae na Floresta Estacional Decidual em Santa Catarina.

Figure 2 - Distribution of the form 7 of rarity of the species of the family Myrtaceae in the Decidous Forest in Santa Catarina.

Diferentemente da condição de vasta distribuição relatada por Legrand \& Klein (1969), esta espécie apresentou distribuição restrita, mas corroborou a descrição de muito rara dos autores, pois quanto ao número de indivíduos apresentou-se escassa (3 indiv.), porém, não teve restrição quanto ao habitat.

A Forma 3 refere-se às espécies cuja distribuição é ampla, no entanto há seletividade por habitat e o número de indivíduos é escasso. Nesta forma foram categorizas duas espécies. Eugenia involucrata foi categorizada como comum na porção meridional da FOD (Caiafa \& Martins 2010), e está presente na FOM e FOD catarinense (Tab. 3). Foi relatada por Legrand \& Klein (1969) como de vasta dispersão no estado, no entanto rara em floresta primária. Eugenia pyriformis aparece em gradiente de raridade mais restritivo para a porção meridional da FOD (Forma 6) e está presente apenas na FOM catarinense (Tab. 3). Legrand \& Klein (1969) consideram esta espécie característica e comum tanto na FOM quanto na FED catarinense, mas neste estudo foi seletiva quanto ao habitat sendo amostrada apenas em formação montana e com escassos indivíduos.

A Forma 2 de raridade foi encontrada apenas para Eugenia rostrifolia. Está distribuída ampla e abundantemente no estado, porém é seletiva quanto ao habitat, ocorrendo apenas em formação montana. Essa espécie foi descrita como característica e de vasta dispersão na FED (Legrand \& Klein 1969). O elevado número de indivíduos amostrados demonstra a importância desta espécie na FED (Tab. 3). No entanto, na Floresta Ombrófila Densa 
foi relatada como rara (Legrand \& Klein 1969), sendo que Caiafa \& Martins (2010) a categorizaram na Forma 4 de raridade, sugerindo uma restrita distribuição nesta floresta (Tab. 3). De fato, quando se amplia a escala de observação essa espécie passa a ter uma distribuição que pode ser considerada restrita, uma vez que ocorre apenas nos estados do Rio Grande do Sul e Santa Catarina (Sobral et al. 2013), mas pelo método e escala metodológica considerada, foi categorizada como de ampla distribuição. Esse resultado também demonstra a sensibilidade do método quando se utiliza limiares subjetivos para as variáveis, sendo que espécies de ampla distribuição, até mesmo para a América do Sul, foram categorizadas com alguma forma de raridade e espécie com restrita distribuição no Brasil, como no caso de Eugenia rostrifolia, foram categorizadas como de ampla distribuição. Ainda assim, a metodologia proposta por Rabinowitz et al. (1986) demonstrou ser aplicável e válida, pois clarifica as prioridades de conservação local.

Destaca-se que a Floresta Estacional Decidual pode apresentar um padrão diferente da distribuição das espécies por esta ser considerada limite da distribuição de muitas espécies (Durigon \& Waechter 2011), fato evidenciado pela presença de poucas espécies de ampla distribuição espacial no Neotrópico (Pennington et al. 2009).

Todas as espécies de Myrtaceae presentes na Floresta Estacional Decidual de Santa Catarina têm dispersão zoocórica, envolvendo vários grupos da fauna como aves, mamíferos, répteis e peixes como espalhadores (Backes \& Irgang 2004; Gressler et al. 2006; Carvalho 2006-2010; Instituto de Botânica de São Paulo 2008). Assim, infere-se que estas espécies, por esta característica, podem ser importantes em projetos de restauração ambiental, uma vez que encontram na fauna associada dispersores naturais de seus propágulos e sementes, podendo colonizar distintas e aleatórias áreas, com aporte na biodiversidade das manchas florestais locais. Sugere-se optar pelo plantio de maior proporção de espécies comuns e diminuir a proporção conforme o gradiente de raridade das espécies. Esta medida pode colaborar para que a restauração aproxime-se da distribuição natural das espécies raras e comuns na natureza (Kageyama \& Lepsch-Cunha 2001). Além disso, espécies de Myrtaceae têm sido relatadas com potencial para uso medicinal, podendo-se destacar Blepharocalyx salicifolius, Campomanesia guazumifolia, $C$. xanthocarpa, Eugenia brasiliensis, E. uniflora e Plinia peruviana (Cruz \& Kaplan 2004), estas presentes na FED catarinense.

Neste contexto, frente às características acentuadas de raridade das espécies de Myrtaceae da Floresta Estacional Decidual em SC apontada pelo método aplicado e na provável manutenção do panorama de degradação relatado para a região, recomenda-se atenção quanto à proteção destas espécies, com ênfase nas espécies categorizadas na Forma 7 de raridade, por serem consideradas as mais vulneráveis desta região fitoecológica.

\section{Agradecimentos}

Os autores agradecem à CAPES, a concessão de bolsa de mestrado em Engenharia Ambiental (FURB); ao Inventário Florístico Florestal de Santa Catarina (IFFSC) e FAPESC - Fundação de Amparo à Pesquisa e Inovação do Estado de Santa Catarina, a cessão dos dados, e à equipe que dele participou.

\section{Referências}

APG - Angiosperm Phylogeny Group. 2009. An update of the Angiosperm Phylogeny Group classification for the order and families of flowering plants: APG III. Botanical Journal of the Linnean Society 161: 105-121.

Backes, P. \& Irgang, B. 2004. Mata Atlântica: as árvores e a paisagem. Paisagem do Sul, Porto Alegre. 396p.

Bergamin, R.S. \& Bodin, C.A. 2006. Composição florística e relações fitogeográficas do componente arbóreo de um fragmento florestal no município de Barra do Ribeiro, Rio Grande do Sul, Brasil. Pesquisas Botânica 57: 217-230.

Broennimann, O.; Vittoz, P.; Moser, D. \& Guisan, A. 2005. Rarity types among plant species with high conservation priority in Switzerland. Botânica Helvetica 2: 95-108.

Caiafa, A.N. \& Martins, F.R. 2010. Forms of rarity of tree species in the southern Brazilian Atlantic rainforest. BiodiversConservation 19: 2597-2618.

Carvalho, P.E.R. 2006. Espécies arbóreas brasileiras. Vol. 2. Embrapa Informação Tecnológica, Brasília; Embrapa Florestas, Colombo. 627p.

Carvalho, P.E.R. 2008. Espécies arbóreas brasileiras. Vol. 3. Embrapa Informação Tecnológica, Brasília; Embrapa Florestas, Colombo. 593p.

Carvalho, P.E.R. 2010. Espécies arbóreas brasileiras. Vol. 4. Embrapa Informação Tecnológica, Brasília; Embrapa Florestas, Colombo. 644p.

Chaves, C.L.; Ferreira, P.I.; Paludo, G.F.; Bernardi, A.P.; Mantovani, A. \& Bortoluzzi, R.L.C. 2009. Espécies nativas de Myrtaceae em fragmentos de Floresta Ombrófila Mista, no município de Ponte Alta, Santa Catarina. Anais do IX Congresso de 
Ecologia do Brasil, 13 a 17 de setembro de 2009, São Lourenço, MG. Pp. 1-3.

Cruz, A.V.M. \& Kaplan, M.A.C. 2004. Uso medicinal de espécies da família Myrtaceae e Melastomataceae no sul do Brasil. Floresta e ambiente 11: 47-52.

Dajoz, R. 1983. Ecologia geral. $4^{\mathrm{a}}$ ed. Vozes, Petrópolis. $472 p$.

Drever, C.R.; Snider, J. \& Drever, M.C. 2010. Rare forest types in northeastern Ontario: a classification and analysis of representation in protected áreas. Canadian Journal of Forest Research 3: 423-435.

Durigon, J. \& Waechter, J.L. (2011). Floristic composition and biogeographic relations of a subtropical assemblage of climbing plants. Biodiversity and Conservation 20: 1027-1044.

Fontana, C. 2012. Formas de raridade da Floresta Estacional Decidual em Santa Catarina: análise de metadados. Dissertação de Mestrado. Universidade Regional de Blumenau, Blumenau. 149p.

Gaston, J. 1994. Rarity. Chapman \& Hall, Londres. 205p.

Gressler, E.; Pizo, M.A. \& Morellato, L.P. 2006. Polinização e dispersão de sementes em Myrtaceae do Brasil. Revista Brasileira de Botânica 4: 509-530.

Instituto de Botânica (IBOT). 2008. Listagem das espécies arbóreas e indicação de sua ocorrência natural nos biomas/ecossistemas e regiões ecológicas do estado de São Paulo, com a síndrome de dispersão, classificação sucessional e a categoria de ameaça de extinção. Disponível em <http://www.ibot.sp.gov. br/pesquisa_cientifica/restauracao_ecologica/ anexo_resol_sma08-08.pdf >. Acesso em 14 agosto 2012.

Inventário Florístico Florestal de Santa Catarina (IFFSC). 2010. Portal do inventário florístico florestal do estado de Santa Catarina. Disponível em <www.iff. sc.gov.br>. Acesso em 15 agosto 2012.

Izco, J. 1998. Types of rarity of plant communities. Journal of Ecology Science 5: 641-646.

Kageyama, P.Y. \& Lepsch-Cunha, N.M. 2001. Singularidade da biodiversidade nos trópicos. In: Garay, I.; Dias, B.F.S.; Garay, I.E.G.; Braulio, F.S. (eds.). Conservação da biodiversidade em ecossistemas tropicais: avanços conceituais e revisão de novas metodologias de avaliação e monitoramento. Vozes, Petrópolis. Pp. 199-214.

Kilka, R.V. \& Longhi, S. J. 2011. A composição florística e a estrutura das florestas secundárias no rebordo do Planalto Meridional. In: Schumacher, M.V.; Longui, S.J.; Brun, E.J. \& Kilka, R.V. (eds.). A Floresta Estacional subtropical: caracterização e ecologia no rebordo do planalto meridional. Pallotti, Santa Maria. Pp. 53-83.

Klein, R.M. 1972. Árvores nativas da Floresta Subtropical do Alto Uruguai. Sellowia, Itajaí 24: 9-62.

Klein, R.M. 1978. Mapa fitogeográfico de Santa Catarina. Flora ilustrada catarinense. Herbário Barbosa Rodrigues, Itajaí. $24 \mathrm{p}$.
Koeppen, W. 1948. Climatologia: con un estudio de los climas de la Tierra. Fondo de Cultura Economica, Ciudad de México. 466p.

Landrum, L.R. \& Kawasaki, M.L. 1997.The genera of Myrtaceae in Brazil: an illustrated synoptic treatment and identification keys. Brittonia 4: 508-536.

Legrand, C.D. \& Klein, R.M. 1967. Mirtáceas: Gomidesia. Flora ilustrada catarinense. Herbário Barbosa Rodrigues, Itajaí. p. 2-44.

Legrand, C.D. \& Klein, R.M. 1969. Mirtáceas: Eugenia . Flora ilustrada catarinense. Herbário Barbosa Rodrigues, Itajaí. Pp. 46-216.

Legrand, C.D. \& Klein, R.M. 1969. Mirtáceas: Myrcia. Flora Ilustrada Catarinense. Herbário Barbosa Rodrigues, Itajaí. Pp. 218-330.

Legrand, C.D. \& Klein, R.M. 1970. Mirtáceas: Myrceugenia. Flora ilustrada catarinense. Herbário Barbosa Rodrigues, Itajaí. Pp. 332-453.

Legrand, C.D. \& Klein, R.M. 1972. Mirtáceas: Calyctorectes. Flora ilustrada catarinense. Herbário Barbosa Rodrigues, Itajaí. Pp. 554-569.

Legrand, C.D. \& Klein, R.M. 1977. Mirtáceas: Campomanesia, Feijoa, Britoa, Myrrhinium, Hexachlamys, Siphoneugena, Myrcianthes, Neomitranthes, Psidium. Flora ilustrada catarinense. Herbário Barbosa Rodrigues, Itajaí. Pp. 572-730.

Legrand, C.D. \& Klein, R.M. 1978. Mirtáceas: Myrciaria [...]. Flora ilustrada catarinense. Herbário Barbosa Rodrigues, Itajaí. Pp. 733-876.

Leite, P.F. \& Klein, R.M. 1990. Vegetação. In: Instituto Brasileiro de Geografia e Estatística. Geografia do Brasil: Região Sul. Fundação Instituto Brasileiro de Geografia e Estatística, Rio de Janeiro. Pp. 113-150.

Lima, D.F.; Goldenberg, R. \& Sobral, M. 2011. O gênero Campomanesia (Myrtaceae) no estado do Paraná, Brasil. Rodriguésia 62: 683-693.

Marchiori, J.N.C. \& Sobral, M. 1997. Dendrologia das angiospermas: Myrtales. UFSM, Santa Maria. 304p.

Nimer, E. 1971. Climatologia da Região Sul do Brasil. Revista Brasileira de Geografia 4: 3-65.

Oliveira-Filho, A.T.; Jarenkow, J.A. \& Rodal, M.J.N. 2006. Floristic relationships of seasonally dry forests of eastern South America based on tree species distribution patterns. In: Pennington, R.T.; Lewis, G.P. \& Ratter, J.A. (org.). Neotropical savannas and dry forests: Plant diversity, biogeography and conservation. CRC Press, Boca Raton. Pp. 151-184.

Pennington, R.T.; Lavin, M. \& Oliveira-Filho, A. 2009. Woody plant diversity, evolution, and ecology in the Tropics: perspectives from seasonally dry tropical forests. Ecology, Evolution and Systematics 40: 437-457.

Pitmann, N.C.A.; Terborgh, J.; Silman, M. R. \& Nuñez, P.V. 1999. Tree species distributions in upper Amazonin forest. Ecology 80: 2651-2661. 
Rabinowitz, D.; Cairns, S. \& Dillon, T. 1986. Seven forms of rarity and their frequency in the flora of the British Isles. In: Soulé, M.E. (ed.). Conservation biology: the science of scarcity and diversity. Sinauer Associates, Sunderland. Pp. 182-204.

Ricklefs, R.E. 2011. A economia da natureza. 6a ed. Guanabara Koogan, Rio de Janeiro. 546p.

Romagnolo, M.B. \& Souza, M.C. 2006. O gênero Eugenia L. (Myrtaceae) na planície de alagável do Alto Rio Paraná, estados de Mato Grosso do Sul e Paraná, Brasil. Acta Botanica Brasilica 20: 529-548.

Schumacher, M.V.; Longhi, S.J.B.; Brun, E.J. \& Kilka, R.V. 2011. A Floresta Estacional Subtropical: caracterização e ecologia no rebordo do planalto meridional. Pallotti, Santa Maria. 320p.

Sobral, M.; Proença, C.; Souza, M.; Mazine, F.; Lucas, E. Myrtaceae. 2013. In: Lista de espécies da flora do Brasil. Jardim Botânico do Rio de Janeiro. Disponível em <http://floradobrasil. jbrj.gov.br/jabot/listaBrasil/ConsultaPublicaUC/ BemVindoConsultaPublicaConsultar.do>. Acesso em 16 setembro 2013.

Stehmann, J.R.; Forzza, R.; Salino, A.; Sobral, M.; Costa, D.P. \& Kamino, L.H.Y. 2009. Plantas da Floresta Atlântica. Jardim Botânico do Rio de Janeiro. 516p.

The Plant List. 2010. A working list of all plant species. Disponível em <http://www.theplantlist.org/>. Acesso em 15 setembro 2013.

Vibrans, A.C. Uhlmann; A. Sevegnani, L.; Marcolin, M.; Nakajima, N.; Grippa, C.R.; Brogni, E. \& Godoy, M.B. 2008. Ordenação dos dados de estrutura da Floresta Ombrófila Mista partindo de informações do inventário florístico-florestal de Santa Catarina: resultados de estudo-piloto. Ciência Florestal 18: 511-523.
Vibrans, A.C.; Sevegnani, L.; Gasper, A.L. \& Lingner, D.V. 2012a. Inventário florístico florestal de Santa Catarina. Diversidade e conservação dos remanescentes florestais. Vol. 1. Edifurb, Blumenau. 344p.

Vibrans, A.C.; Sevegnani, L.; Gasper, A.L. \& Lingner, D.V. 2012b. Floresta Estacional Decidual. Vol. 2. Edifurb, Blumenau. 336p.

Vibrans, A.C.; Sevegnani, L.; Gasper, A.L. \& Lingner, D.V. 2013a. Floresta Ombrófila Mista. Vol. 3. Edifurb, Blumenau. 440p.

Vibrans, A.C.; Sevegnani, L.; Gasper, A.L. \& Lingner, D.V. 2013b. Floresta Ombrófila Densa. Vol. 4. Edifurb, Blumenau. 576p.

Vibrans, A.C.; Sevegnani, L.; Uhlmann, A.; Schorn, L.A.; Marcolin, M., Sobral, M.G.; Gasper, A.L.; Lingner, D.V. \& Bonnet, A. 2011. Inventário florístico florestal de Santa Catarina. Relatório final. Vol. 1-11. Universidade Regional de Blumenau, Blumenau. 2315p. (mimeo).

Vibrans, A.C.; Sevgnani, L.; Lingner, D.V.; Gasper, A.L. de \& Sabbagh, S. 2010. Inventário florístico florestal de Santa Catarina (IFFSC): aspectos metodológicos e operacionais. Pesquisa Florestal Brasileira 30: 291-302.

Vieira, F.C.S. \& Quadros, K.E. de. 2010. Myrtaceae, Myrcia squamata (Mattos and D. Legrand) Mattos and Myrceugenia seriatoramosa (Kiaersk.) D. Legrand and Kausel in Santa Catarina: distribution extension. Check List 6: 488-490.

Vieira, F.C.S. 2010. Myrtaceae Juss. no alto Quiriri, Garuva, Santa Catarina, Brasil. Dissertação de Mestrado. Universidade de São Paulo, São Paulo. 80p.

Vitali, M. \& Uhlig, V.M. 2010. Unidades de Conservação de Santa Catarina. Sustentabilidade em Debate 1: 43-61. 